\title{
CORONOMICS AND SOCIOECONOMIC ASPECTS OF THE ENERGY SECTOR
}

\author{
DAVIT NARMANIA \\ Doctor of Economics, Professor, \\ Ivane Javakhishvili Tbilisi State University, Georgia \\ davit.narmania@tsu.ge
}

Abstract. The worldwide pandemics of COVID-19 has influenced all sectors of the economy. Energy sector as one of the branches of the economy has been affected as well. Suspension of a number of enterprises and institutions, switching staff to the remote working mode and other restrictions for the purpose of preventing risks stemming from the pandemics reduces overall energy consumption. On the other hand, staying at home significantly increases household energy consumption. However, the reduction of the energy consumption due to the stop/less active operation of the enterprises and institutions usually exceeds the amount of increased household consumption. Subsequently, amount of the energy to be distributed is reduced.

Another important factor is the price of energy. Energy price in Georgia is mainly determined according to the exchange rate of the national currency in relation to US Dollar. Pandemics and decrease of economic activities have caused dramatic drop of Georgian Lari rate. This has increased price of both - imported energy (electricity and natural gas) and electricity to be purchased from the local hydro power plants on the basis of the power purchase agreements so called PPAs (where the purchase price of the electricity is indicated in US Cents).

During the pandemics the solvency of enterprises/organizations and the population is also quite important. Restrictions related to pandemics have reduced revenues of business sector as well as of the population. Many enterprises and institutions (e.g. hotels, restaurants, cafes, beauty parlors etc.) have completely stopped. Hereby, a lot of employees have lost their jobs and the income or the remuneration for some people has decreased due to pandemics. Therefore, many subscribers have faced problem of paying bills and applied companies as well as the regulatory commission and the State with request to defer their debts. Envisaging social background, the decision of the Government of Georgia to subsidize utility services of the natural persons definitely bears quite progressive character. The Government of Georgia has spent more than 170 million GEL within 3 months for those subscribers whose monthly electricity consumption doesn't exceed $200 \mathrm{~kW}$ and natural gas consumption doesn't exceed 200 cubic meters. For such consumers the Government has also subsidized water and cleaning service fees.

This article analysis abovementioned and other socioeconomic aspects of the energy sector that have become relevant due to COVID-19.

KEYWORDS: CORONOMICS; ENERGY; CONSUMPTION; UTILITY SUBSIDIES.

For citation: Narmania, D., (2020). Coronomics and Socioeconomic Aspects of the Energy Sector. Globalization and Business, 10. 58-62. https://doi.org/10.35945/gb.2020.10.006 


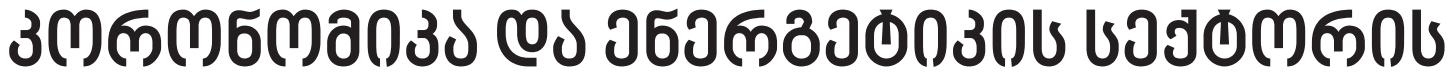

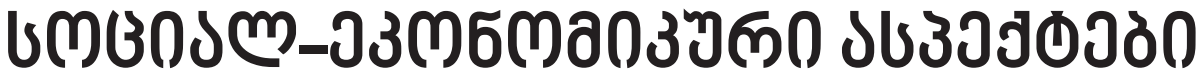

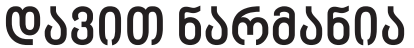

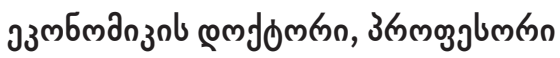

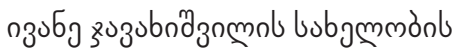

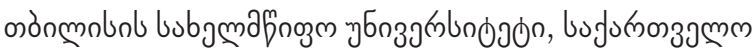

davit.narmania@tsu.ge

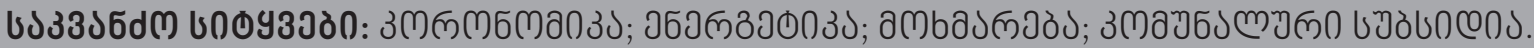

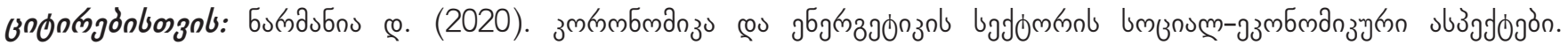

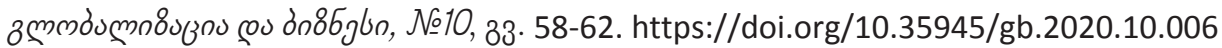

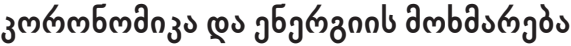

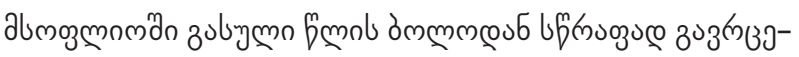

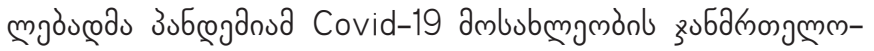

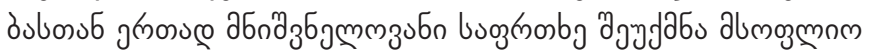

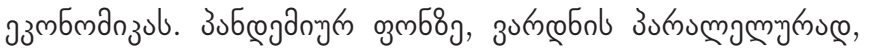
పьмозмпм ззмбмдаз

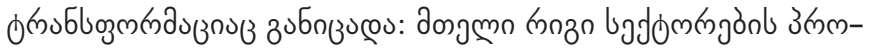

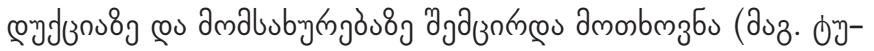

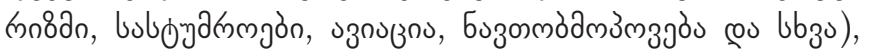

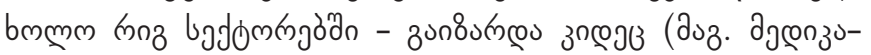

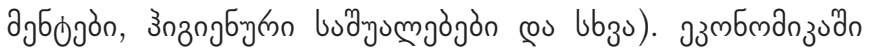

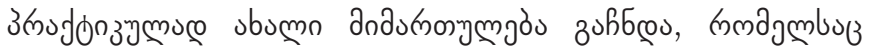

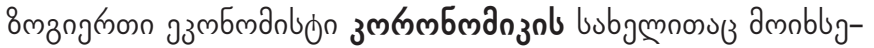

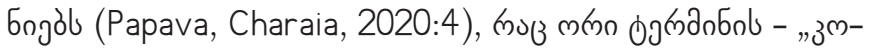

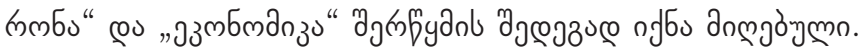

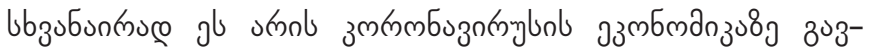
mgbnl gybfosmu (Ikechukwu, Tochukwu, 2020:2). gkno-

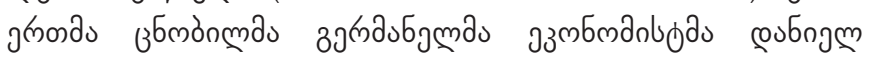

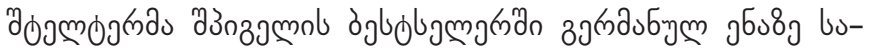

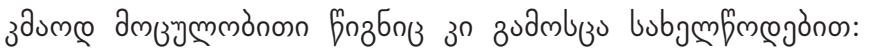
Coronomics/Nach dem Corona-Schock: Neustart aus der

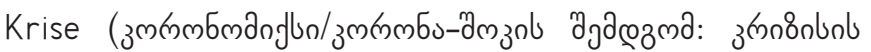
bymubumn coubufynobn) (Coronomics, 2020). उubeganol

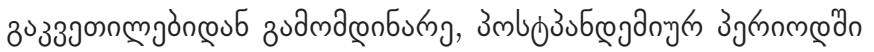
ambummm об

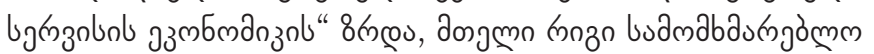
bufmbmal jmadd 2020:12).

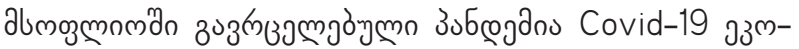

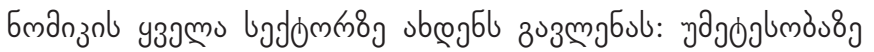

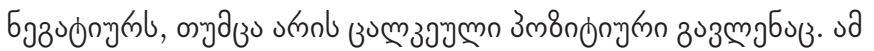

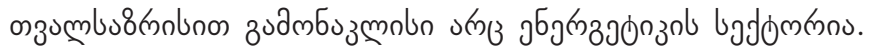

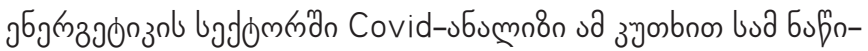

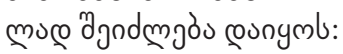

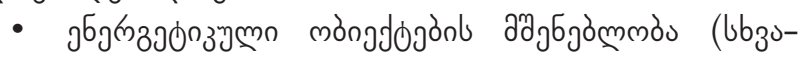

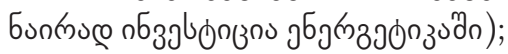

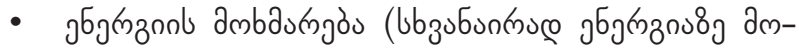
мbmз 50$)$;

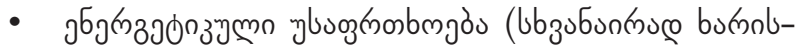

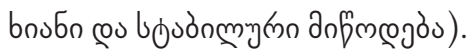

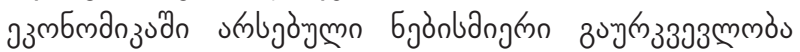
(aubibu8

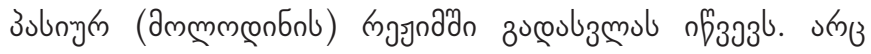
grnon nб

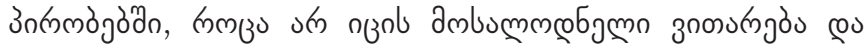

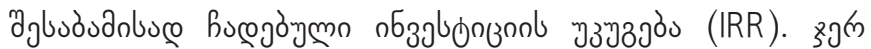

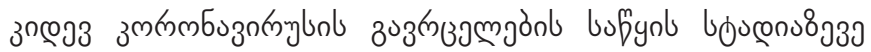

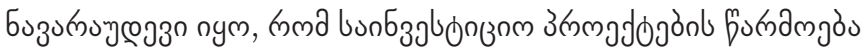

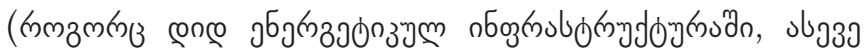

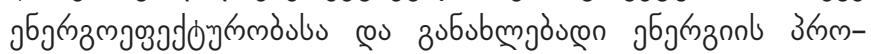

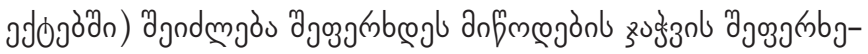

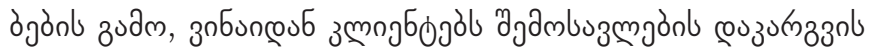

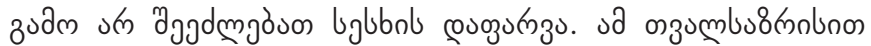

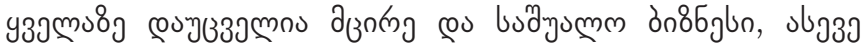

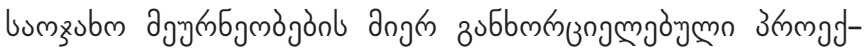

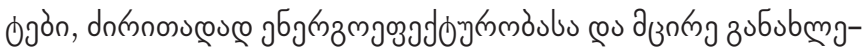

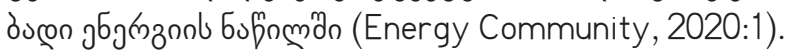

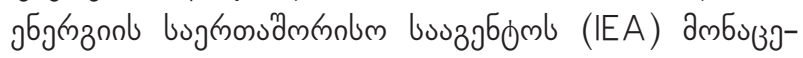

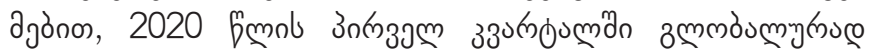

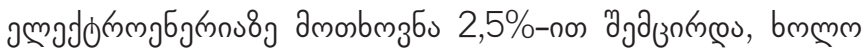

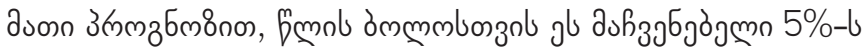

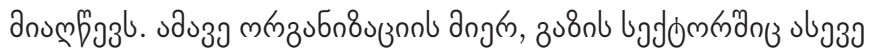

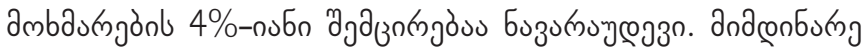




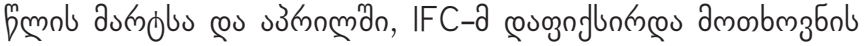

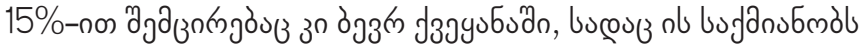
(The Impact of Covid-19, 2020:2).

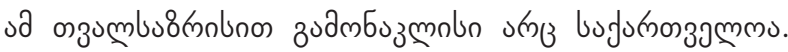

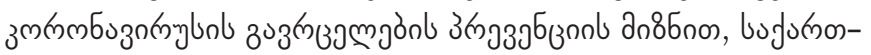

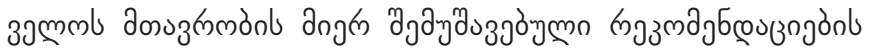

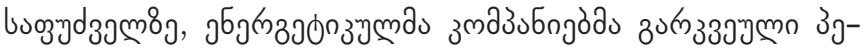

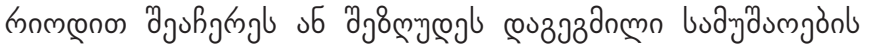

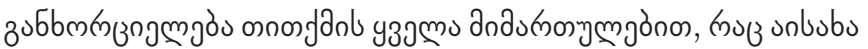

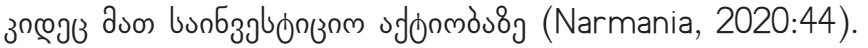

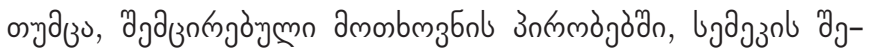

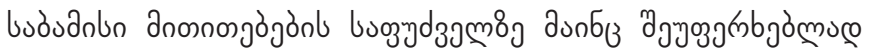
sbeg

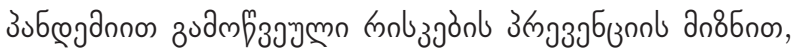

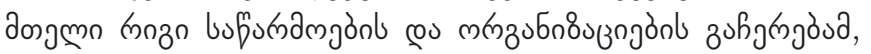

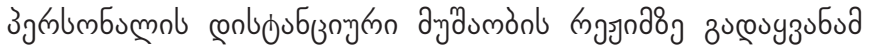

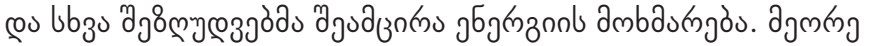

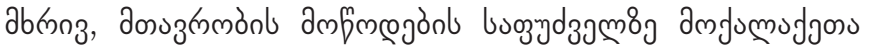

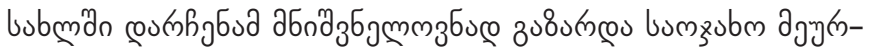

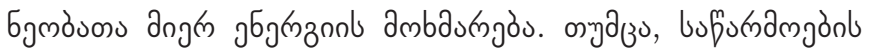

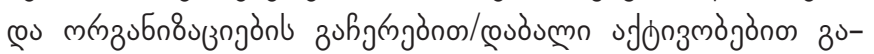

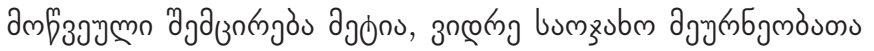

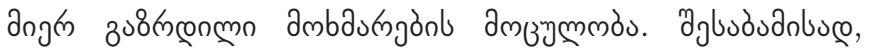

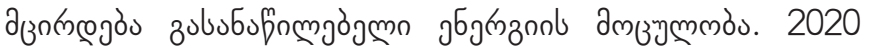

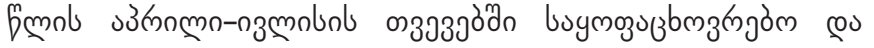

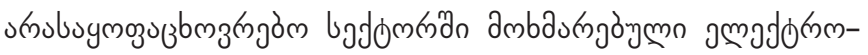

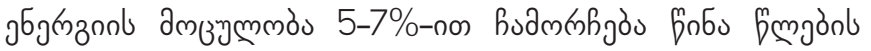

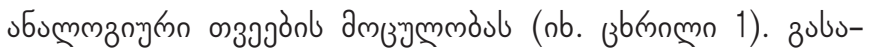

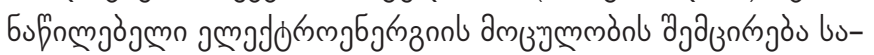

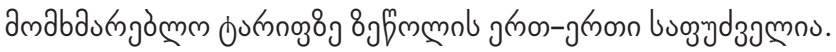

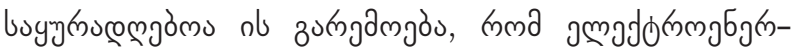

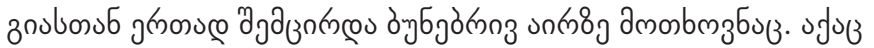

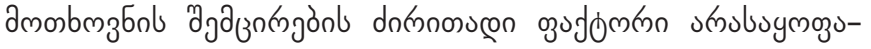

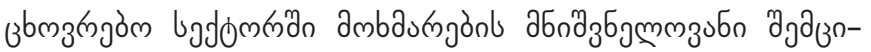

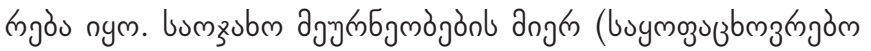

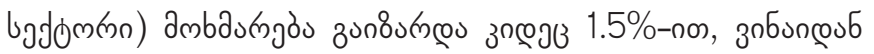

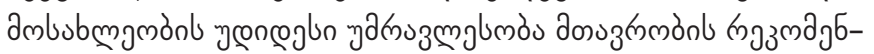

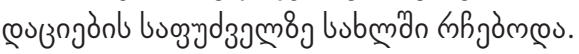

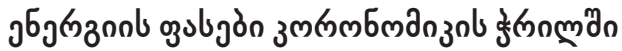

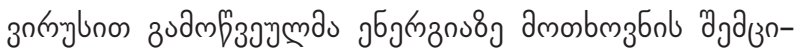

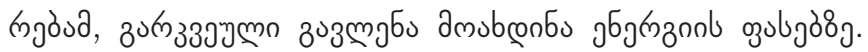

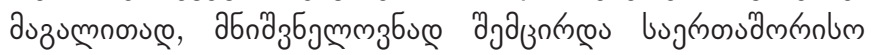

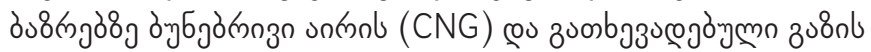

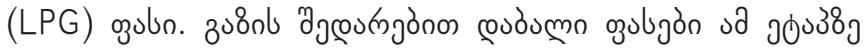

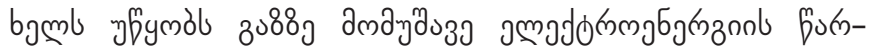

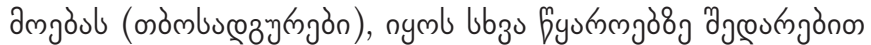

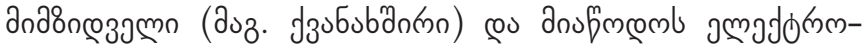

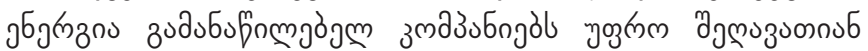
gुoluce (The impact of Covid-19,2020).

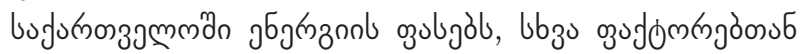

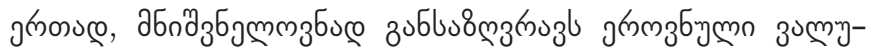

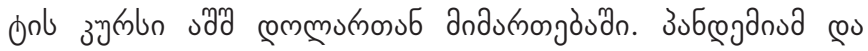

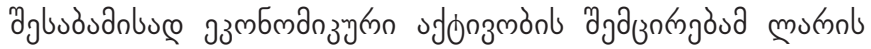

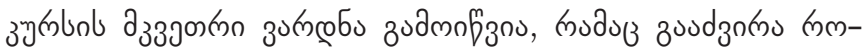

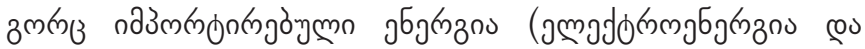

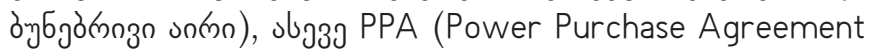

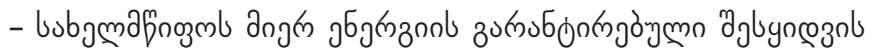

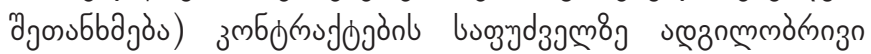

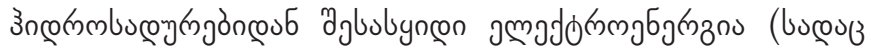

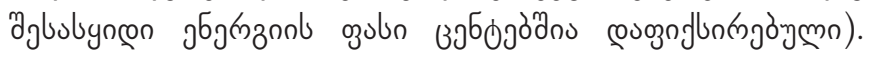

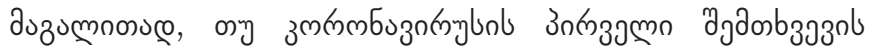

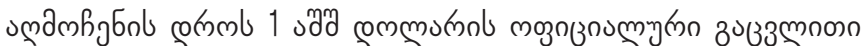

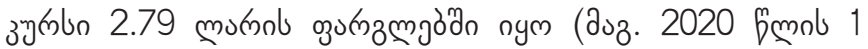

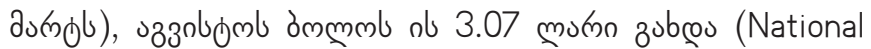

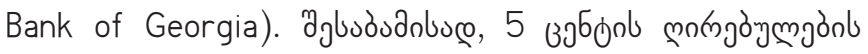

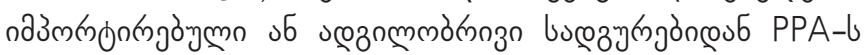

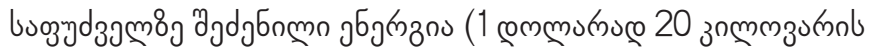

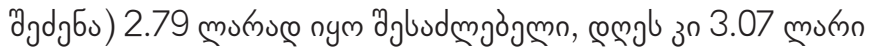

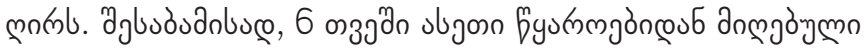

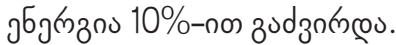

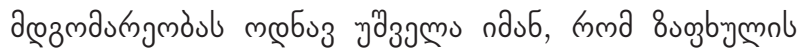

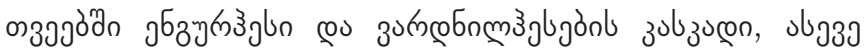

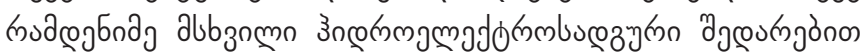

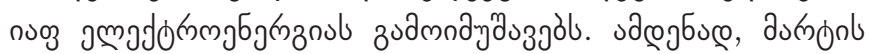

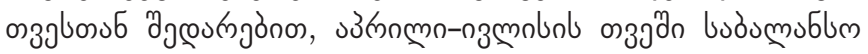

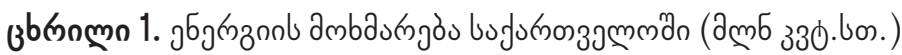

\begin{tabular}{|l|r|r|r|r|r|r|r|}
\hline 2020 & 1217.878 & 1149.234 & 1050.333 & 947.106 & 957.299 & 995.956 & 993.746 \\
\hline 2019 & 1148.418 & 1148.418 & 1142.304 & 1018.839 & 1117.984 & 1117.984 & 1164.845 \\
\hline 2018 & 1169.701 & 1072.399 & 1114.259 & 1040.991 & 1161.168 & 1175.209 & 1224.575 \\
\hline 2017 & 1097.590 & 1029.722 & 1024.652 & 938.983 & 1061.721 & 1101.854 & 1208.246 \\
\hline
\end{tabular}

fyurnm: gluzm, https://esco.ge/ka/energobalansi/by-year- 


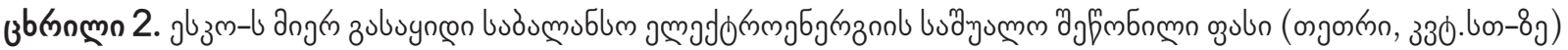

\begin{tabular}{|c|c|c|c|c|c|}
\hline зупомео & 2016 & 2017 & 2018 & 2019 & 2020 \\
\hline оsб3зйо & 13.4270458 & 13.6486315 & 12.802977 & 13.5254479 & 14.5868671 \\
\hline 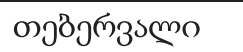 & 13.6103928 & 13.5322024 & 13.102101 & 13.6543756 & 13.8837893 \\
\hline పుణ்ం & 8.2133089 & 12.6371223 & 12.2154634 & 13.4438273 & 18.4660737 \\
\hline ১зஙলmo & 10.5698605 & 11.3560223 & 12.1184845 & 12.9927551 & 16.0888149 \\
\hline asobo & 10.2519912 & 9.4280967 & 13.2595499 & 7.3628251 & 13.2076731 \\
\hline o3бolo & 10.6248766 & 12.1870003 & 12.6449173 & 7.2278252 & 13.1741005 \\
\hline o3molo & 9.3008829 & 11.9140872 & 8.8884730 & 13.6034633 & 15.0346724 \\
\hline s33olsిm & 10.0003359 & 11.1646907 & 11.9046817 & 14.2842237 & \\
\hline 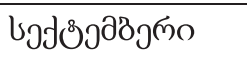 & 10.1199242 & 11.7757291 & 11.6111821 & 14.8374457 & \\
\hline мపతీмдరృణం & 11.0193625 & 13.7681373 & 14.0393031 & 14.6874306 & \\
\hline бмлдљупо & 10.6789665 & 14.1366798 & 13.8013228 & 15.4506703 & \\
\hline ๔эзјдљјюо & 11.2303984 & 12.6636783 & 14.0379822 & 14.729727 & \\
\hline
\end{tabular}

fyurnm: jluzm, https://esco.gelelectricity/balancing-electricity-price

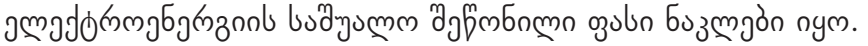

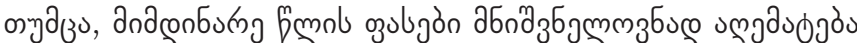

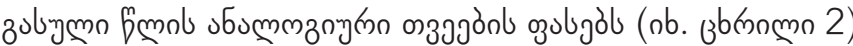
(ESKO).

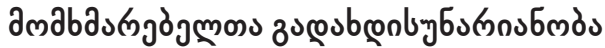

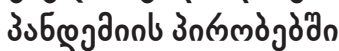

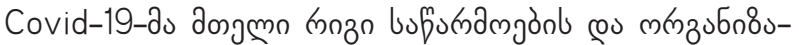

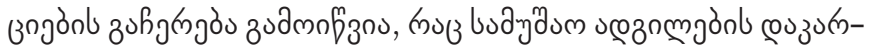

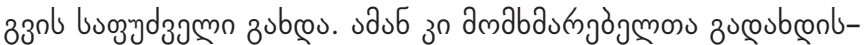

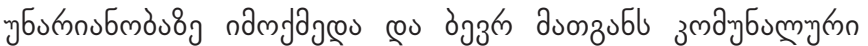

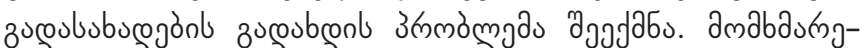

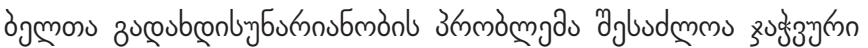

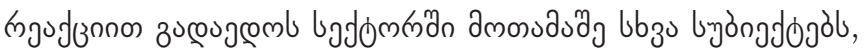

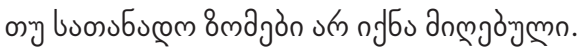

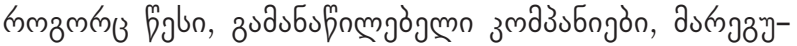

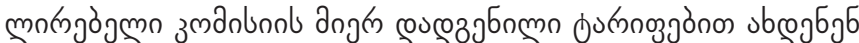

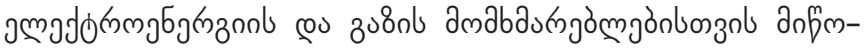

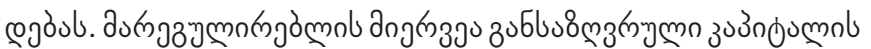

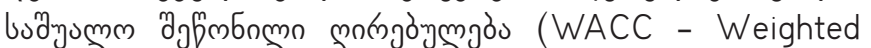

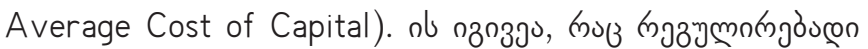

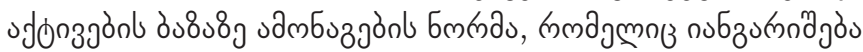

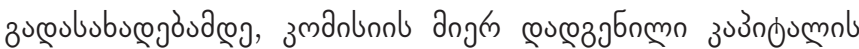

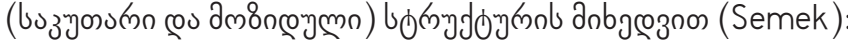

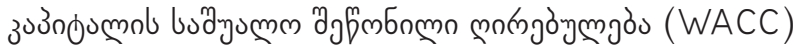

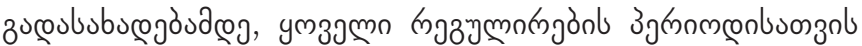

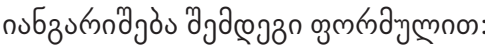

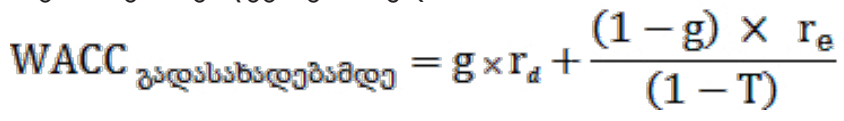

bocoos:

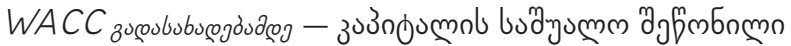

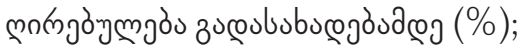

$G$ - bgubol fnemo (\%);

rd - bgbbolu mongòngmgòs (\%);

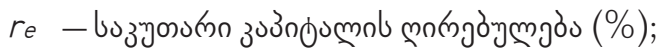

$T$ - amzjònl zucoububucon (\%).

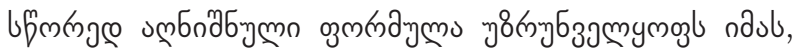

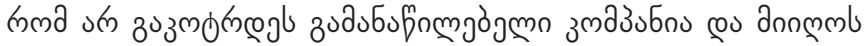

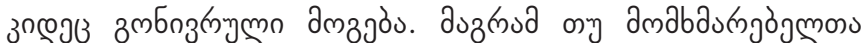

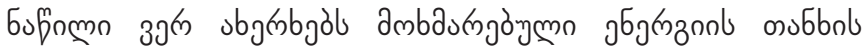

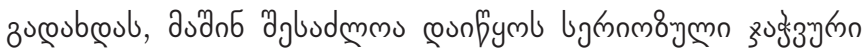

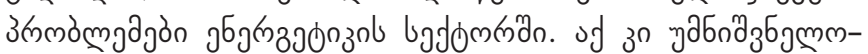

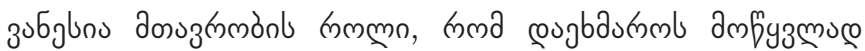

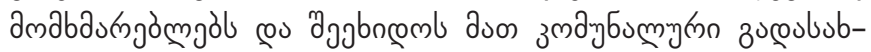
cogmgònl zucoubcougn.

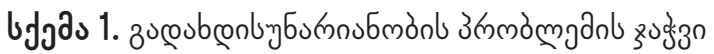

\begin{tabular}{|c|c|c|c|c|}
\hline 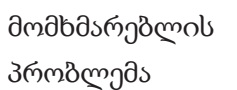 & 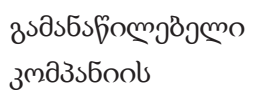 & 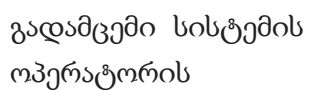 & 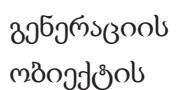 & 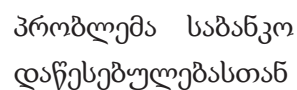 \\
\hline & उґмдвмлаs & 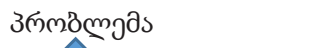 & З๓мдемлаs & \\
\hline
\end{tabular}




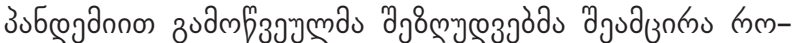

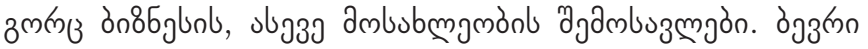

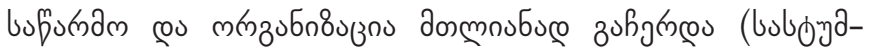

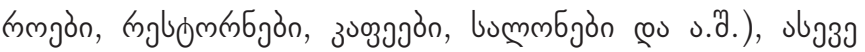

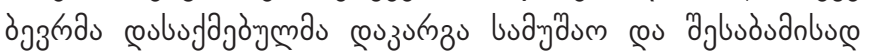

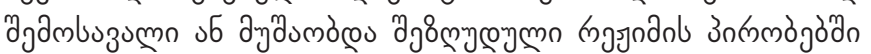

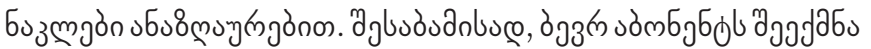

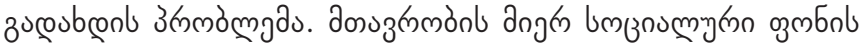

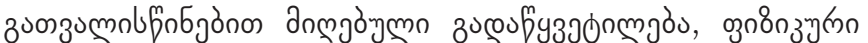

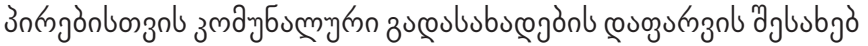
youzme 3

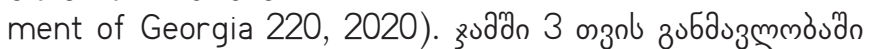

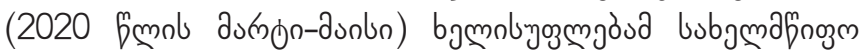

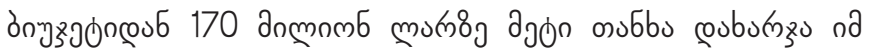

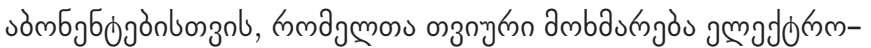

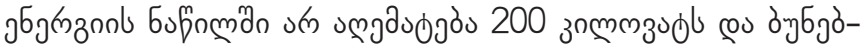

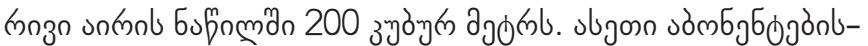

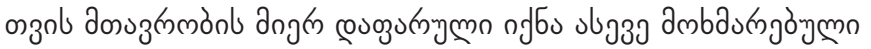

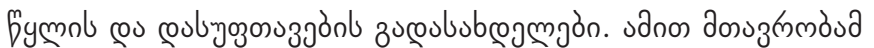

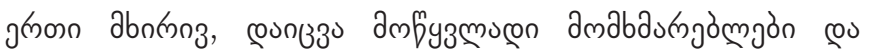

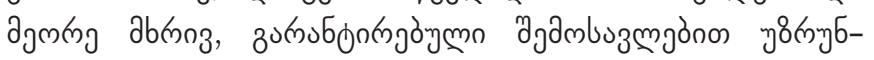

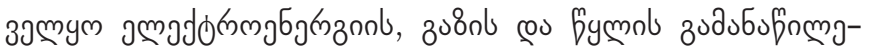

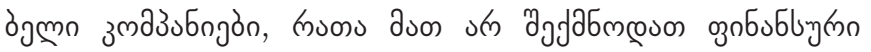
उ ऊmmòm

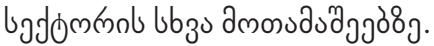

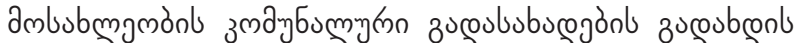

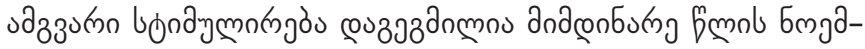

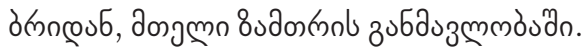

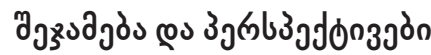

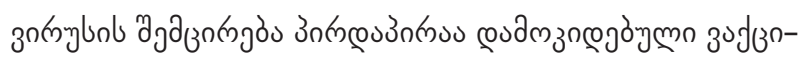

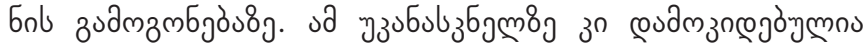

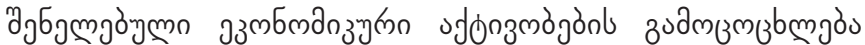

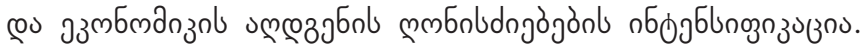

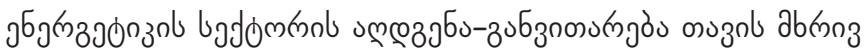

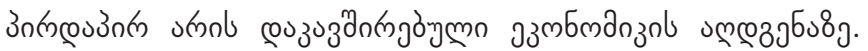

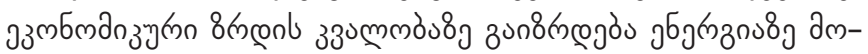

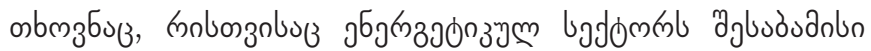

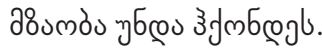

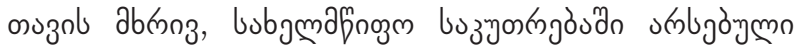

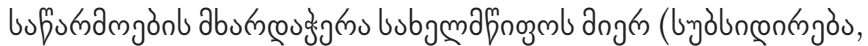

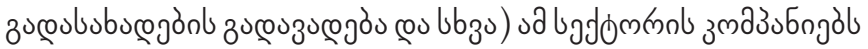

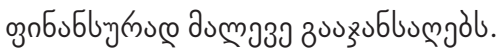

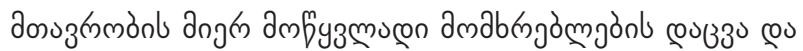

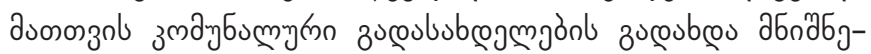

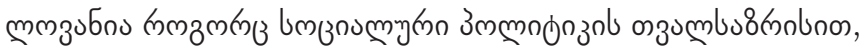

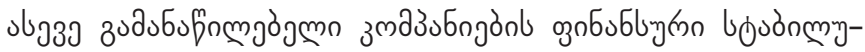
mmónbonzol.

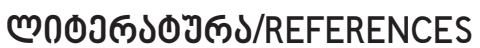

Aptsiauri, D., (2020). Current Trends in the Development of Modern International Economic Relations and the Post-Crisis Challenges of the Pandemic (COVID-19), Georgian Strategy and International Relations Research Foundation, \# 139. (In Georgian)

Narmania, D., (2020). COVID-19, Energy and Investment, Journal. Forbes, April 2020. (In Georgian)

Papava, V., \& Charaia, V., (2020). The Economic Crisis and Some Challenges of the Georgian Economy, Georgian Strategy and International Relations Research Foundation, \# 136. (In Georgian)

Resolution \# 220 of the Government of Georgia of April 3, 2020 «On Approval of the Rules and Conditions for Subsidizing Utility Taxes», (In Georgian).

General Recommendations for New Coronavirus (SARS-CoV-2) Infection (COVID-19) for Electricity Supply, Water Collection / Treatment / Distribution and Waste Management, Annex \# 10, IDPs from the Occupied Territories, Labor, Health and Social Welfare of Georgia Ministry, 2020. (In Georgian)

2019 Report of the Georgian National Energy and Water Regulatory Commission. (In Georgian) www.gnerc.org

Commercial energy system operator, (In Georgian) www.esco.ge

De, A., (2020). The energy sector post COVID-19: refueling the recovery, KPMG, June 2020.

Ikechukwu, E., \& Tochukwu, V. (2020). Coronomics and global economy: A purview of the impact of Coronavirus on the global economy, (2020).

Ghiani, E., Galici, M., Mureddu, M., \& Pilo, F., (2020). Impact on Electricity Consumption and Market Pricing of Energy and Ancillary Services during Pandemic of COVID-19 in Italy, Energies 2020, 13, 3357.

Energy investments and financing during and post COVID-19 crisis, Energy Community, April 2020.

IAEE Energy Forum, Special COVID-19 Edition, Third Quarter 2020, ISSN 1944-3188.

The impact of COVID-19 on Energy Market Stability, IEF Insight Brief, April 2020.

The impact of COVID-19 on the Power Sector, IFC, June 2020. 